\title{
Aplicaciones de la química física a la tecnología de alimentos. Motivación al estudio y desarrollo de competencias transversales
}

\author{
Fernando Cardona ${ }^{\text {a, b }}$, Concepción Rubio-Granero ${ }^{\text {b,c }}$ \\ ${ }^{a}$ Departamento de Tecnología de los Alimentos. Escuela Técnica Superior de Ingeniería Agronómica y del Medio \\ Natural. Universitat Politècnica de València. \\ ${ }^{b}$ Unitat de Genètica Molecular. Instituto de Biomedicina de Valencia. CSIC \\ ${ }^{\circ}$ Centro Integrado Público de Formación Profesional Vicente Blasco Ibáñez de Valencia
}

\begin{abstract}
Resumen
Es conocido que el aprendizaje basado en problemas y el aprendizaje en contexto mejoran el proceso enseñanza-aprendizaje, especialmente en asignaturas complejas y en las que es difícil ver su aplicación práctica. Esto puede ser debido a que mejora la motivación del alumno, además de permitir introducir una actividad de aprendizaje activo.

Por este motivo, por tercer año, consecutivo se ofreció la posibilidad de realizar trabajos sobre aplicaciones de la asignatura al futuro campo laboral, incluyendo también ejemplos durante las lecciones magistrales. Además, corrigiendo las cuestiones que en años anteriores han sido observadas como negativas en esta actividad (que los trabajos sean en inglés y el nivel de dificultad para alumnos de primero), se utilizaron actividades en castellano y más sencillos, introduciendo también trabajos de fin de grado, de máster y libros. Las encuestas realizadas posteriormente muestran que se resuelven estos problemas, y al alumno no le resulta tan arduo realizar esta actividad.
\end{abstract}

Los resultados académicos muestran también que las calificaciones y el número de aprobados mejoran de forma significativa en los alumnos que realizan las tareas, probablemente debido a que se toma consciencia de la utilidad y la importancia del conocimiento de esta materia en la tecnología de alimentos.

Además, las encuestas realizadas muestran que estos trabajos resultan útiles para el desarrollo y la evaluación de cuatro competencias transversales: comprensión e integración; pensamiento crítico; comunicación efectiva; y trabajo en equipo y liderazgo.

Palabras clave: Aprendizaje en contexto, Aprendizaje basado en problemas, Motivación, Competencias transversales. 
Aplicaciones de la química fisica a la tecnología de alimentos. Motivación al estudio y desarrollo de competencias transversales.

\section{Introducción}

La Química Física es percibida como una materia compleja por los alumnos de grados que no son de los campos de Química, Física o Ingeniería (Mulop et al., 2012; Sokrat et al., 2014). Esto es debido fundamentalmente a su complejidad matemática y a la aparente falta de relación con el futuro campo de trabajo en dichos grados. El problema puede ser que se enfatiza demasiado en los métodos matemáticos, sin dejar claro el significado de los conceptos, y dificultando que pueda identificarse su aplicación en situaciones reales (Flores y Trejo, 2003; Durán-Aponte y Durán-García, 2013).

El Aprendizaje en Contexto (AC) Basado en Proyectos (ABP) ha demostrado ser muy útil y apropiado en el proceso de enseñanza-aprendizaje de materias complejas (De Jong, 2006). El enfoque contextualizado, además de suponer una motivación extra para el alumno, sirve para introducirlo en su campo de trabajo mediante ejemplos reales, aplicando así los conceptos teóricos impartidos en el aula (Woodburn, 1977; Eilks y Hofstein, 2015). El AC produce mejores resultados, especialmente en asignaturas complejas, o cuando resulta difícil ver su actividad laboral (Pinto y Martín, 2012). El ABP es una metodología de aprendizaje activo en la que el alumno se convierte en protagonista de su aprendizaje, trabajando en pequeños grupos tutorizados por el profesor (Villalobos-Delgado et al, 2016). La resolución de problemas puede usarse como base para alcanzar objetivos de aprendizaje y desarrollar competencias relacionadas con la futura actividad laboral. Esta metodología cambia el rol del alumno a un papel activo, responsable y autónomo; y también el del profesor, actuando como apoyo y guía, sin tener el papel central. Además, permite integrar conocimientos de diferentes áreas, por lo que se aproxima mucho más a la vida real que otras metodologías de enseñanza (Rodríguez-Sandoval et al., 2010). El ABP se ha descrito además como una metodología desarrolladora de competencias transversales o genéricas (Olivares-Olivares y Heredia-Escorcia, 2012; Cardona y Atarés, 2017). También se ha postulado que el proceso de enseñanza-aprendizaje de la química se ve favorecido en un contexto de formación por competencias laborales, comparándolo con la metodología tradicional (Cejas-Yanes y Castaño-Oliva, 2014).

Por estos motivos, en este proyecto se introdujeron en 2015 en la asignatura "Fundamentos Químicos para la Ciencia y Tecnología de Alimentos", en la Unidad Didáctica 2 (UD2, Química Física), ejemplos relacionados con la Ciencia y Tecnología de Alimentos (CTA) durante las clases, así como la posibilidad de realizar trabajos voluntarios relacionados con estas aplicaciones. Además, la actividad puede llevarse a cabo en grupos, permitiendo así el aprendizaje cooperativo, que ha demostrado su eficacia para el aprendizaje de esta materia y otras (Méndez-Coca, 2012), y el desarrollo de la competencia de trabajo en grupo. La realización de la tarea supone para el alumno una aproximación de la asignatura al campo de trabajo de su grado, ayudando al entendimiento de los conceptos teóricos y motivando al alumno a su estudio (Cardona y Atarés, 2016). 
Para evaluar el resultado de la introducción de esta metodología docente, se analizaron varios ítems mediante encuestas al alumnado. También se compararon las calificaciones obtenidas por los alumnos que habían realizado el trabajo y los que no. Los resultados de los tres cursos, muestran que tienen un efecto positivo en la nota final y en el desarrollo de competencias transversales, pero que los alumnos lo perciben como una tarea compleja y no les gusta demasiado realizarla. A algunos alumnos también les cuesta presentarlo en persona, por lo que prefieren hacerlo mediante herramientas de screencast.

\section{Objetivos}

El objetivo general de este trabajo es determinar si la introducción de ejemplos reales tiene un efecto positivo en el proceso de enseñanza aprendizaje. Para ello, este objetivo se subdivide en tres objetivos parciales:

1-Evaluar la introducción del AC-ABP en la UD2 de la asignatura "Fundamentos Químicos para CTA", incorporando la posibilidad de realizar trabajos voluntarios (para subir nota) sobre aplicaciones de la materia a la CTA.

2-Evaluar el impacto sobre la motivación de los alumnos en el estudio de la asignatura y sus beneficios en el proceso de enseñanza-aprendizaje, evaluando los siguientes ítems:

-Interés por la actividad

-Beneficios generados de su realización

-Efecto sobre las calificaciones finales obtenidas

3-Evaluar la utilidad de esta actividad para el desarrollo y evaluación de CTs.

\section{Desarrollo}

Esta experiencia se llevó a cabo con alumnos de primer curso del grado de CTA (Escuela Técnica Superior de Ingeniería Agronómica y del Medio Natural), de la Universitat Politècnica de València (UPV). La asignatura es anual de 12 créditos repartidos en tres UDs.

Desde el curso académico 2014-2015 se introdujeron ejemplos reales de aplicaciones de la materia al futuro campo laboral de los estudiantes (AEC). Además, se planteó a los alumnos la posibilidad de realizar trabajos sobre aplicaciones de la Química Física a la 
Aplicaciones de la química física a la tecnología de alimentos. Motivación al estudio y desarrollo de competencias transversales.

CTA (AC-ABP). Los trabajos son voluntarios y sirven para subir la nota final de la UD2 hasta un punto. Los alumnos tienen la posibilidad de hacerlo individual o en grupo (hasta un máximo de 3 participantes), así como de exponer el trabajo en clase o hacerlo en vídeo mediante herramientas de screencast, recomendándose la herramienta ScreenCast-OMagic, que permite visualizar al alumno mientras expone y dispone de una versión gratuita. En el caso de utilizar screencast, el vídeo generado se sube al repositorio de la UPV (https://media.upv.es/\#/portal), por lo que está accesible a los compañeros, y además se proyecta en el aula. La exposición comprende un máximo de 10 minutos (a dividir entre varios alumnos si el trabajo se realiza en grupo) y en cualquier caso (en persona o screencast), después de la exposición se abre una discusión del trabajo con los compañeros y el profesor. Por último, se utiliza la evaluación por pares, empleando para evaluar los trabajos una rúbrica proporcionada por el profesor. Con una encuesta posterior se mide la percepción de los alumnos acerca de estas metodologías, así como los beneficios que aportan a su formación y al desarrollo de CTs. Para realizar la encuesta y analizar los resultados se utilizó la herramienta correspondiente de Google Drive (Formularios de Google). El efecto sobre la nota final alcanzada se evaluó mediante la herramienta Excel de Microsoft Office.

\section{Resultados y discusión}

\subsection{Comparativa de las calificaciones obtenidas}

Para determinar si la incorporación del ABP mejoraba las calificaciones de los alumnos, se compararon las notas obtenidas en el examen de teoría (TA), problemas (PROB) y la nota final, sin tener en cuenta la calificación extra obtenida por otras actividades (incluida ésta).

Los resultados obtenidos (Fig. 1) muestran que los alumnos que realizan el ABP obtienen mejores calificaciones globales de manera estadísticamente significativa, y que ese aumento de la nota es debida a la parte del examen que corresponde con la resolución de problemas. No se observan diferencias en las calificaciones obtenidas en la TA.

Estos resultados son similares a los obtenidos en cursos anteriores (Cardona y Atarés 2016; Cardona y Atarés 2017), lo que confirma que la metodología es adecuada para mejorar el proceso enseñanza-aprendizaje en esta materia, al mejorar las calificaciones en la parte aplicada del examen. 


\subsection{Interés en la realización de los proyectos de aplicaciones de la materia a la CTA}

Del total de alumnos examinados (91), 78 (85,7\%) participaron voluntariamente en el AC-ABP y de éstos 42 (53,8\%) realizaron la encuesta. De los 42 encuestados, 29 (69\%) realizaron la presentación por screencast, y el resto lo expusieron en clase. Los porcentajes que utilizaron screencast sobre el total son similares a los de los encuestados, siendo la muestra representativa de la población en este ítem.

En la Fig. 2 se muestran las respuestas de los 42 alumnos encuestados en cuanto al disfrute realizando el proyecto. Los porcentajes muestran que la mayoría $(40,5 \%)$ es neutral, pero hay más alumnos a los que gusta poco o nada $(35,7 \%)$ que a los que gusta bastante o mucho (23,8\%). Estos resultados indican en general poco aceptación, posiblemente debido a que se trata de una tarea difícil para alumnos de primer curso, como se ha observado con anterioridad (Cardona y Atarés, 2017). Por ese motivo, aunque se ha bajado la dificultad de los trabajos con respecto a años anteriores, parece que es importante seguir buscando trabajos de más fácil comprensión a estos niveles, para que el esfuerzo a realizar por el alumno sea adecuado a sus conocimientos.

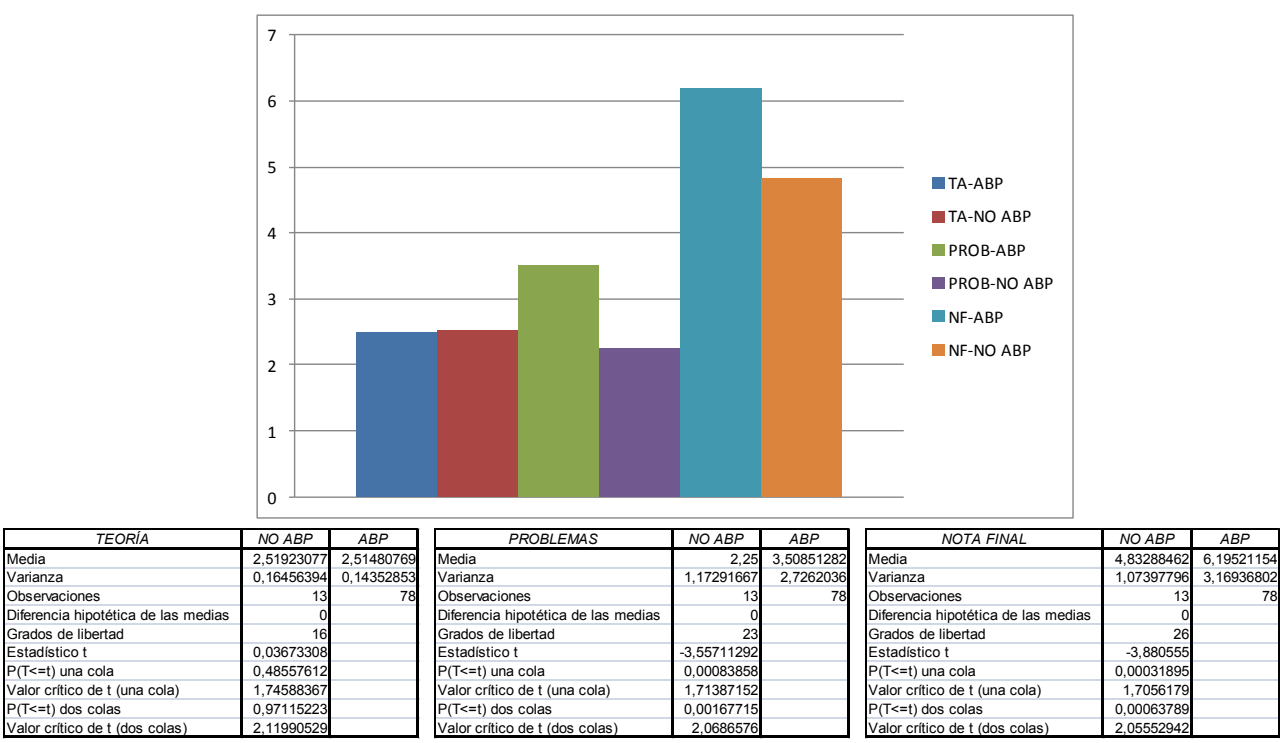

Fig. 1. Distribución de las medias de las calificaciones en función de la elaboración o no del proyecto de ABP.

Se muestran las diferencias obtenidas en la parte de teoría (TA), problemas (PROB) y nota final (NF).

Las diferencias son estadísticamente significativas en un test $t$-student para varianzas desiguales para

NF y PROB $(\mathrm{p}<0,05)$ (ver tablas en la parte inferior de la figura). No se observan diferencias en las calificaciones obtenidas en la TA. Fuente: Elaboración propia.

En cuanto a los motivos por los que no exponen los proyectos en clase y utilizan Screencast-O-Magic, se muestran en la Fig. 3, siendo el principal motivo el miedo a hablar 
Aplicaciones de la química fisica a la tecnología de alimentos. Motivación al estudio y desarrollo de competencias transversales.

en público $(55,2 \%)$, seguido por la intención de ahorrar tiempo en el aula $(27,6 \%)$ y la curiosidad por aprender a utilizar la herramienta de presentación en vídeo $(24,1 \%)$.

Los resultados muestran que el miedo a hablar en público es el principal problema para la realización de la tarea, y quizá sea uno de los motivos de la falta de aceptación de la misma (Fig.1). Por este motivo, quizá sea recomendable no dar la opción de hacerlo en vídeo, ya que esta opción parece más fácil de realizar en general, y más sencilla de desarrollar por ellos mismos en cualquier momento que sea necesaria. En cualquier caso, parece una actividad adecuada para el desarrollo de la CT "Comunicación efectiva" en ambos formatos.

\section{En general ¿te ha gustado hacer el trabajo?}
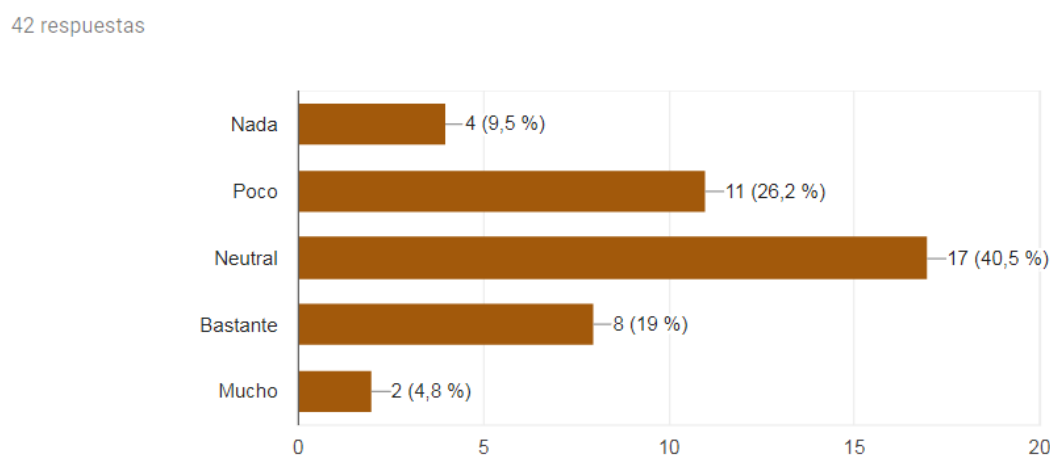

Fig. 2. Interés por la realización de los trabajos de aplicaciones. Se muestra el porcentaje de alumnos que elige cada respuesta. Los resultados muestran que al 35.7\% de los alumnos les disgusta hacer el trabajo, frente a un $23.8 \%$ que les gusta. Fuente: Elaboración propia.

Si elegiste presentarlo por ScreenCast, fue por:

29 respuestas

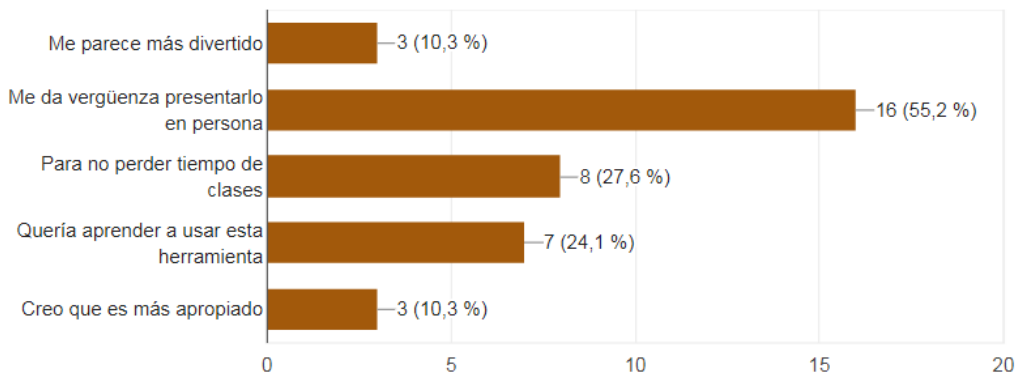

Fig. 3. Motivos para no exponer los trabajos en clase y utilizar ScreenCast-O-Magic. Se muestra el porcentaje de alumnos que elige cada respuesta. Los resultados muestran que el $55.2 \%$ de los alumnos eligieron este método por miedo a hablar en público, y solo un $10.3 \%$ porque les gusta más. Fuente: Elaboración propia. 


\subsection{Desarrollo y evaluación de CTs}

Como ya se ha comentado en el apartado anterior, la actividad parece adecuada para desarrollar y evaluar la CT “Comunicación efectiva”, al tener que exponerse el trabajo, bien en persona o bien en vídeo. Además, la actividad parece a priori también adecuada para desarrollar las CTs "Comprensión e integración", al tener que comprender e integrar los conceptos teóricos en un proyecto real; "Aplicación y pensamiento práctico" por los mismos motivos que la anterior; "Trabajo en equipo y liderazgo", ya que es una actividad que se realiza en grupos de 2-3 personas; "Planificación y gestión del tiempo", ya que el tiempo de exposición es limitado; y “Conocimiento de problemas contemporáneos”, ya que se analizan aplicaciones a problemas reales y actuales. También podría servir para desarrollar otras, aunque de una manera más superficial, como son "Diseño y proyecto" ya que se expone un proyecto como si fuera propio; y "Pensamiento crítico", ya que se pide un análisis crítico del trabajo expuesto.

Por este motivo, se pidió en la encuesta a los alumnos que indicaran que CTs les ayudaba a desarrollar esta actividad (Fig. 4), incluyendo en la encuesta una rúbrica de evaluación de CTs y el vídeo explicativo de la UPV (http://www.upv.es/contenidos/COMPTRAN/info/1026833normalc.html). Las CTs definidas por la UPV son:

1-Comprensión e integración; 2-Análisis y resolución de problemas; 3-Diseño y proyecto; 4-Responsabilidad ética, medioambiental y profesional; 5-Pensamiento crítico; 6Aprendizaje permanente; 7-Instrumental específica; 8-Aplicación y pensamiento práctico; 9-Innovación, creatividad y emprendimiento; 10-Trabajo en equipo y liderazgo; 11Comunicación efectiva; 12-Conocimiento de problemas contemporáneos; 13-Planificación y gestión del tiempo

Los resultados (Fig. 4) muestran que la opinión de los alumnos es similar a la de los profesores, ya que entienden que la actividad les sirve sobre todo para desarrollar las CTs "Comunicación efectiva", "Trabajo en equipo y liderazgo", "Diseño y proyecto", “Comprensión e integración” y "Conocimiento de problemas contemporáneos". En menor grado, también les parece útil para desarrollar las CTs "Planificación y gestión del tiempo". "Innovación, creatividad y emprendimiento" y "Pensamiento Crítico". Sin embargo, y esto sí discrepa de la opinión de los profesores, no les parece tan útil para desarrollar las CT "Aplicación y pensamiento práctico" y "Aprendizaje permanente", probablemente debido a diferencias en el concepto que define esta CT. 
Aplicaciones de la química fisica a la tecnología de alimentos. Motivación al estudio y desarrollo de competencias transversales.

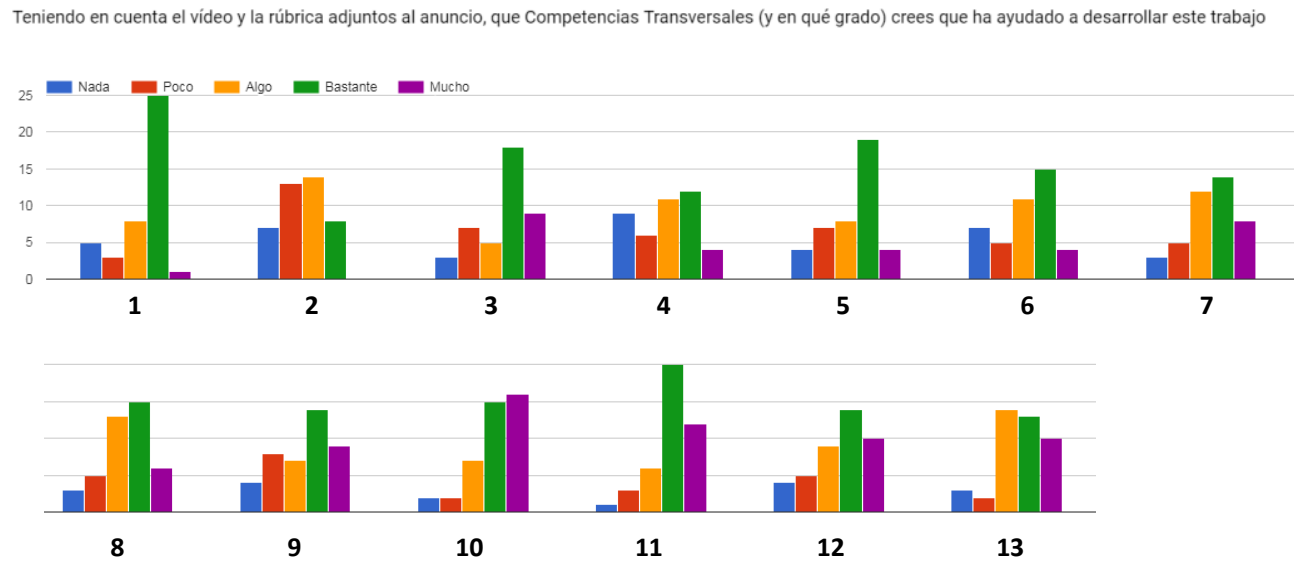

Fig. 4. Percepción de los alumnos respecto al desarrollo de CTs según la lista de la UPV (1-Comprensión e integración; 2-Análisis y resolución de problemas; 3-Diseño y proyecto; 4-Responsabilidad ética, medioambiental y profesional; 5-Pensamiento crítico; 6-Aprendizaje permanente; 7-Instrumental específica; 8-Aplicación y pensamiento práctico; 9-Innovación, creatividad y emprendimiento; 10-Trabajo en equipo y liderazgo; 11Comunicación efectiva; 12-Conocimiento de problemas contemporáneos; 13-Planificación y gestión del tiempo). Se observa que los alumnos opinan que ayuda a desarollar, de más a menos sumando los votos de las opciones "bastante" y "mucho" (se indica entre paréntesis la suma del número de votos), las CTs 11 (32); 10 (31); 3 (27); 1

(26); 12 (24); 5,9 y 13 (23), 2 y 7 (22), 8 (21); y por último la 6 (19). Fuente: Elaboración propia.

Además, cabe decir aquí que esta actividad se utilizó, junto a otras evidencias obtenidas en la asignatura, para evaluar una de las CTs de la que la asignatura es punto de control, "Conocimiento de problemas contemporáneos".

En general, los resultados muestran que la actividad se percibe como útil, tanto por los profesores como por los alumnos, para el desarrollo y evaluación de varias CTs, por lo que se propone implantar la actividad también para este fin.

\subsection{Percepción de los beneficios aportados por la realización de los proyectos de aplicaciones en cuanto a la calificación final y percepción general de la actividad.}

Para conocer la percepción de los alumnos en cuanto a lo que modifica la calificación final, se incluyo en la encuesta una pregunta a ese respecto (Fig. 5). Los resultados muestran que de forma muy generalizada (aproximadamente el 74\%) consideran que el efecto sobre la nota final les parece apropiado. Además, también la mayoría de los encuestados (69\%) recomiendan hacer el trabajo (Fig.6), por lo que en estos aspectos no parece apropiado hacer modificaciones en la actividad. 


\section{Conclusiones}

La introducción del AC-ABP, introduciendo trabajos sobre aplicaciones de la materia en el campo laboral y ejemplos reales durante las clases de aula:

1-La introducción del AC-ABP en la asignatura mejora las calificaciones de los alumnos en la parte de problemas del examen.

2-No se percibe en general como una tarea agradable, ya que a la mayoría de encuestados les es indiferente o no les gusta.

3-La exposición en el aula presenta como problema el miedo a hablar en público.

4-Se percibe que aporta beneficios claros en el desarrollo de varias CTs.

5-En general los alumnos consideran apropiado la influencia de la actividad a la calificación, y recomiendan a otros alumnos realizarla.

\section{La influencia del trabajo sobre la nota final crees que es:}

42 respuestas

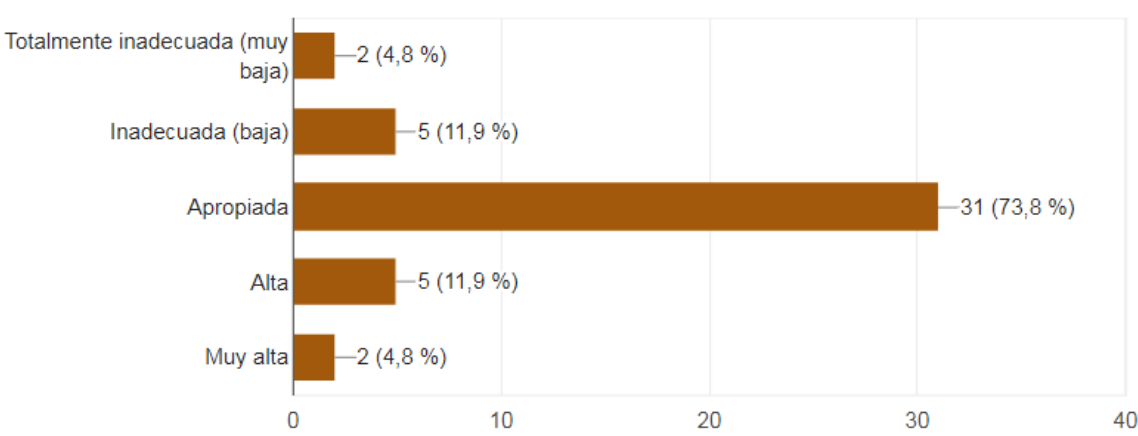

Fig. 5. Percepción de la influencia de la actividad sobre la nota final. Se muestra el porcentaje de alumnos que vota cada opción. Los resultados muestran que la mayoría de los alumnos (73.8\%) cree que la contribución en la calificación es apropiada. Fuente: Elaboración propia. 


\section{En general, ¿recomendarías hacer el trabajo?}

\section{2 respuestas}

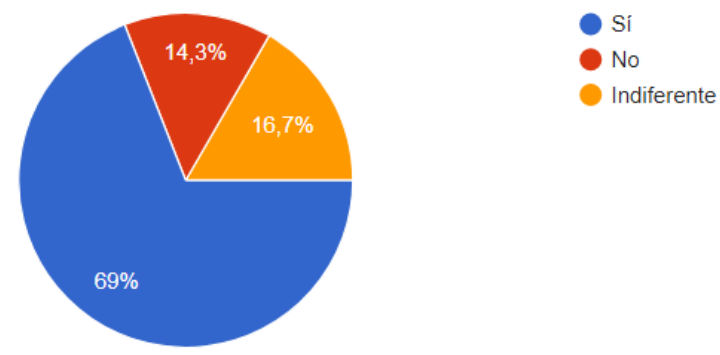

Figura 6. Percepción general de la realización del trabajo. . Se muestra el porcentaje de alumnos que vota cada opción. Los resultados muestran que la mayoría de los alumnos (69\%) recomendaría a otros alumnos realizar la actividad. Fuente: Elaboración propia.

\section{Perspectivas}

A la vista de los resultados, parece interesante seguir con la metodología iniciada, si bien convendría corregir algunos defectos detectados en los proyectos propuestos, como sigue siendo el elevado nivel de los conceptos tratados y el esfuerzo que necesita emplear el alumno para realizarlos. Además, cabe plantearse si hacer todas las presentaciones por ScreenCast para que aprendan a manejar esa herramienta, o todo lo contrario, exigir que se expongan los trabajos en clase para que se pierda el miedo a hablar en público Los ejemplos introducidos durante las clases de aula parecen del nivel correcto.

\section{Referencias}

Cardona, F.\& Atarés, L. (2016). Motivación al estudio de la Fisicoquímica en el Grado de Ciencia y Tecnología de Alimentos mediante el estudio de aplicaciones. IN-RED 2016.

Cardona, F. \& Atarés, L. (2017). Introducción de aplicaciones de la termodinámica a la tecnología de alimentos como elemento motivador y de aprendizaje en contexto. INNODOCT 2017.

Cejas-Yanes, E. \& Castaño-Olivas, R. (2004) La formación química en un contexto de competencias laborales. Revista Electrónica de Enseñanza de las Ciencias. 3,2: 171-189

De Jong, O. (2006). Context-based chemical education: how to improve it? Plenary lecture presented at the 19th International Conference on Chemical Education. Seoul, Korea, 12-17 August 2006. 
Durán-Aponte, E. \& Durán-Gracía, M. (2013). Aprendizaje cooperativo en la Enseñanza de Termodinámica: Estilos de Aprendizaje y Atribuciones Causales. Revista Estilos de Aprendizaje $11: 11$

Eilks, I. \& Hofstein A. (2015) Relevant Chemistry Education: From Theory to Practice. Rotterdam: Sense publishers.

Flores, S. \& Trejo, L. (2003). ¿Cómo Mejorar el Proceso Enseñanza - Aprendizaje Mediante la Evaluación - Regulación? El Caso de la Termodinámica. Memorias de las Terceras Jornadas Internacionales de la Enseñanza Universitaria de la Química. La Plata, Argentina. 28 Septiembre al 1 de octubre 2003.

Mendez-Coca, D. (2012). Motivational Change Realized by Cooperative Learning Applied in Thermodynamics. European Journal of Physics Education 3(4): 13-26, 2012

Mulop, N., Yusof, K. M. \& Tasir, Z. (2012). A review on enhancing the teaching and learning of thermodynamics. Procedia-Social and Behavioral Sciences, 56, 703-712.

Olivares-Olivares S. L. \& Heredia-Escorcia, Y. (2012). Revista mexicana de investigación educativa. 17,54: $759-778$

Pinto, G. \& Martín, M. (2012). Enseñanza y Divulgación de la Química Física. Madrid: Garceta.

Rodríguez-Sandoval, E., Vargas-Solano, E. M. \& Luna-Cortés, J. (2010). Evaluación de la estrategia de aprendizaje basado en proyectos. Educación y educadores, 13,1: 13-25.

Sokrat, H., Tamani, S., Moutaabbid M. \& Radid, M. (2014). Difficulties of Students from the Faculty of Science with Regard to Understanding the Concepts of Chemical Thermodynamics. Procedia Social and Behavioral Sciences. 116, 21: 368-372.

Villalobo-Delgado, V., Ávila-Palet J. E. \& Lizett-Olivares S. (2016). Aprendizaje basado en problemas en química y el pensamiento crítico en secundaria. Revista Mexicana de Investigación Educativa, 21,69: 557-581.

Woodburn, J.H. (1977) Using applied chemistry to tackle motivation problems. Journal of. Chemistry Education. 54,12: 763 